\title{
Environment Monitoring System Raising Awareness of Sensitive Inhabitants Based on Regression Predictive Modelling
}

\author{
Razvan BOGDAN ${ }^{\mathrm{a}, 1}$, Mihaela CRIȘAN-VIDA ${ }^{\mathrm{a}}$, Razvan MURESAN ${ }^{\mathrm{a}}$, Darius \\ BARMAYOUN $^{\mathrm{b}}$ and Lăcrămioara STOICU-TIVADAR ${ }^{\mathrm{a}}$ \\ ${ }^{a}$ University Politehnica Timisoara, Faculty of Automation and Computers, Timișoara, \\ Romania \\ ${ }^{\mathrm{b}}$ University Politehnica Timisoara, Research Center in Engineering and Management, \\ Timișoara, Romania
}

\begin{abstract}
The system described in this paper notifies the user about possible indoor environmental hazards through a smartphone application. Events such as house fires or increasing levels of dangerous substances are detected; thus, raising the awareness level in regard to the quality of the indoor environment. Our solution is a system that monitors the environment within a room in order to avoid undesired health hazards. It consists of a data collection subsystem, a cloud database which stores the collected information and a mobile application that displays and notifies the user in case of critical events that might negatively affect the health condition.
\end{abstract}

Keywords. IoT, monitoring, cloud, sensors, indoor air quality

\section{Introduction}

The importance of monitoring the indoor air quality (IAQ) by each individual has been highlighted by the ongoing COVID-19 pandemic. Spending more time at home, even while working, emphasized the need of a cheap but reliable system that is capable of detecting any unwanted event that might negatively affect the inhabitants' health condition due to an increased level of dangerous substances.

This paper aims to develop an affordable, DIY system that can be used on a daily basis by an individual to be notified of any possible hazards through a smartphone application. Moreover, the system is to be developed with customization in mind, in order to adapt the solution to one's need.

\section{Related work}

Current researches underline the importance of monitoring indoor air quality as humans spend most of their time in indoor environments. The rapid advancement of the Internet

${ }^{1}$ Corresponding Author, Razvan Bogdan, University Politehnica Timisoara, Romania, Faculty of Automation and Computers, Bd. Vasile Parvan no.2, Timisoara, Romania; E-mail: razvan.bogdan@cs.upt.ro. 
of Things (IoT) technology and the development of affordable sensors transformed IAQ measurement systems, making them cheaper and smarter [1, 2, 3, 4].

Addressing the importance of the IAQ of public institutions during the COVID-19 outbreak, Mumtaz et al. [5] present a solution for monitoring and predicting the IAQ. Several sensors record data related to eight different pollutants along with temperature and humidity. By applying machine learning algorithms to the recorded data, the IAQ prediction reached a precision of $99 \%$ and an accuracy of $99.37 \%$. Also, the importance of long-term IAQ monitoring in such cases is highlighted by references [2,6].

Another important IAQ related health issue is tackled by Cho [7]. Indoor smoking activity detection is implemented using both analytical and machine learning approaches. The paper implements an environmental sensor fusion approach that successfully detects indoor smoking.

Using Wireless Sensor Networks (WSN) Gonzalez et al. [8] create a low-cost, lowpower, multi-purpose device able to measure the IAQ and detect gas leakage in real-time. The availability and scalability of the system allow users to register nodes while the system is running. Furthermore, a graphene sensor is developed to provide an accurate NO2 measurement while making the data available on a web-based platform.

The modularity of a system using WSN is also proven by the work of Benammar et al. [9]. The authors make use of a WSN to measure the IAQ by focusing on the temperature, humidity, and other six different gases.

Taştan and Gökozan [4] adopt a do-it-yourself (DIY) and open-source approach to monitoring the air quality, allowing individuals to easily implement such a system for their living environment. Various sensors are used to collect data which is further monitored by a Wi-Fi controller and a free to use IoT platform. The resulting data of the paper show that natural ventilation significantly improves the air quality in one's home.

Following a similar DIY approach Salamone et al. [7] describe the results of an open-source project aiming to measure the indoor environmental quality (IEQ). A lamp is transformed into a so-called "smart lamp" by adding a CO2 concentration sensor and a lux sensor.

Similarly, Marques and Pitarma [2] develop a low-cost, real-time system capable of detecting various gases and displaying the data through a smartphone application. The mobile application is also notifying the user of real-time low IAQ levels to ventilate the space or deactivate pollutant-producing equipment.

Rajkumar and et. al [10] describe a home appliance automation system which uses different sensor to monitor and control the house.

\section{Materials and methods}

Our solution consists of a data collection subsystem, a cloud database capable of storing the collected data and a mobile application that displays and notifies the user in case of critical events such as undetected house fires or alarming levels of dangerous chemicals. 


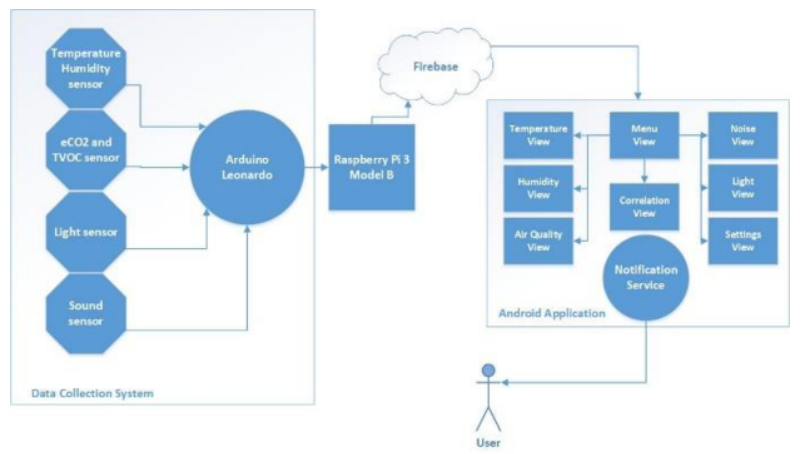

Figure 1. System Architecture

An Arduino board receives environment data from humidity, temperature, light, eCO2 (equivalent Carbon Dioxide), TVOC (Total Volatile Organic Compounds) and noise sensors. The received information is then processed by averaging out the sampled values and further forwarded to the Raspberry Pi 3 through Universal Serial Bus (USB). The Arduino software samples the sensors every second and provides every 30 seconds the averaged values as input for the Raspberry device.

Raspberry Pi 3 takes care of formatting and uploading the data to Firebase. It is backed up by a power bank that is able to supply power to the system even while it is charging itself, acting just like an Uninterruptible Power Supply (UPS). The system stores the data locally and in case of Firebase connection failure, it will upload all the waiting data list when the connection is reestablished.

The Android application displays all the environmental parameters read by the sensors within activities. Each parameter activity displays a plot containing the last 12 hours of sampled sensor data, together with the actual, minimum, maximum, and averaged value. A short prediction is then displayed based on the best fit line of the last 5 minutes or its equivalent of 10 data points. The equation is further used to plot the next 10 data points for the corresponding time interval. The data is also interpreted by showing if one parameter value is increasing or decreasing based on the slope of the best fit line equation.

Correlation view displays the correlation scatter plot between two selected parameters containing the last 12 hours of data. A regression best fit line is drawn together with the equation of the line, the Pearson's $r$ coefficient, which measures how strong the correlation between the two selected variables is.

The user can configure the notifications by customizing the thresholds. The notifications are displayed once the last data point is outside the defined limits. The notification service runs in background and it uses less system resources, so the battery level will not be affected. It listens to events triggered on the Firebase database (such as a new value being added) and determines which notification to display through the smartphone application.

By interpreting the data and correlating temperature and TVOC, the application can determine if there is a house fire. This can be the case if both temperature and TVOC parameters are rapidly increasing and are strongly correlated.

The fire event detection works by correlating the temperature and VOC values and by monitoring the increase of the parameters. The regression best fit line is used to estimate how much the temperature and VOC values will increase in the future. If the temperature is projected to be over 50 degrees Celsius, VOCs are projected to be over $3000 \mathrm{ppb}$ and both are correlated, the user will be notified for a possible house fire event. 
The Arduino code was written using Arduino IDE while the Python scripts were written using the text editor from Raspberry Pi Linux OS.

\section{Results}

Figure 2 shows the parameters measured by the system: temperature, humidity, light activity, noise, and air quality.

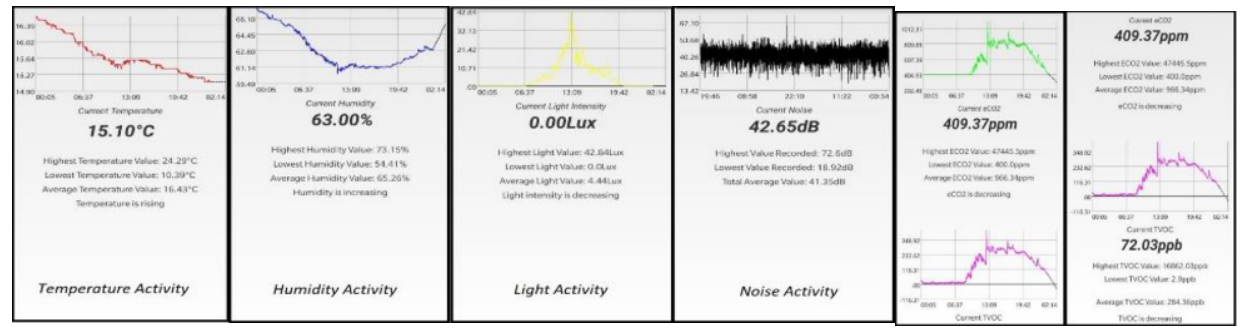

Figure 2. Parameters measure by the system

Figure 3 presents the Smartphone application interface which allows the users to edit notification thresholds and status.
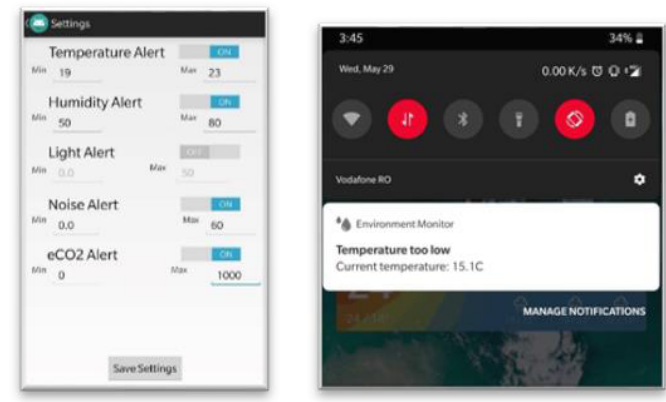

Figure 3. User interface for editing and receiving notifications

Figure 4 presents the data collection and experimentation. A strong correlation was noticed between the $\mathrm{CO} 2$ and VOC values.

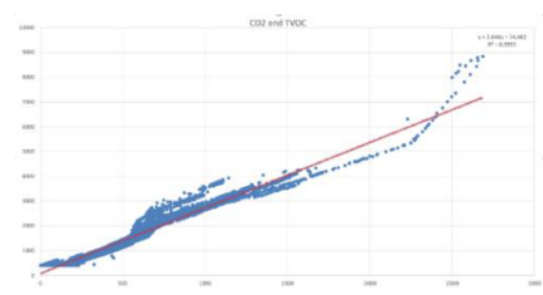

Figure 4. Correlation between eCO2 and TVOC

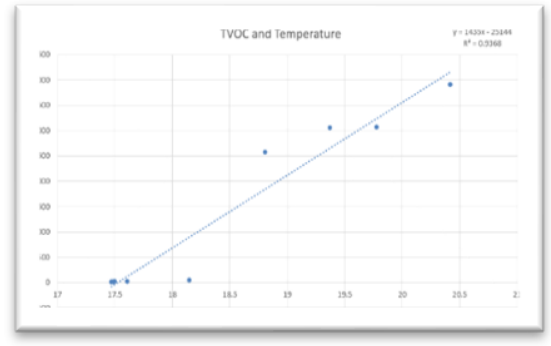

Figure 5. Correlation between TVOC and temperature during the 5-minute fire event window

The graph presented in Figure 4, shows the strongly correlated parameters. If one of the two changes, the other one will most likely change in the same degree. $R$. In the above presented case, it is around 0.95 with the line equation being $y=2.64 x+74.5$. 
When simulating a house fire, a correlation between temperature and TVOC (Figure 5) could be observed. When a fire is inside the room, the temperature and air pollution rapidly rise. This correlation was used to detect such events by computing the best fit line equation every time a new data point was added. In Figure 5 can be observed during 5 minutes how the parameters are correlated, the TVOC and the temperature. By firstly correlating the two parameters, a strong correlation is detected (no correlation is detected if there is no fire). The projected value can be checked using the regression best fit line for temperature and TVOC and further verify if the projected points are within the safe threshold (35 degrees Celsius for temperature and 1000ppb TVOC concentration).

\section{Conclusions}

An IoT system capable of monitoring the environment within a living area was developed. Indoor living environments must be monitored in order to avoid any unwanted events that might negatively affect the inhabitants' health condition due to an increased level of dangerous substances. The solution presents the collected data through a smartphone application and notifies the user in case of abnormal values.

Furthermore, a strong correlation between the temperature and TVOC parameters has been observed when simulating a house fire. Even a small number of parameters, such as 10 data points are enough to determine a possible house fire by computing the best-fit line equation.

The presented solution is a prototype aiming to improve the IAQ within an indoor living space. Therefore, the quality of the sensors limits the reliability and accuracy of the collected data. Moreover, the architecture must be improved by reducing its complexity. This will increase the system's scalability, allowing it to be implemented it in crowded indoor public spaces.

\section{References}

[1] Kim J, Hwangbo H. Sensor-Based Optimization Model for Air Quality Improvement in Home IoT. Sensors. 2018;18(4):959.

[2] Marques G, Pitarma R. A Cost-Effective Air Quality Supervision Solution for Enhanced Living Environments through the Internet of Things. Electronics. 2019;8(2):170.

[3] Salamone F, Belussi L, Danza L, Galanos T, Ghellere M, Meroni I. Design and Development of a Nearable Wireless System to Control Indoor Air Quality and Indoor Lighting Quality. Sensors. 2017;17(5):1021.

[4] Taştan M, Gökozan H. Real-Time Monitoring of Indoor Air Quality with Internet of Things-Based ENose. Applied Sciences.2019;9(16):3435.

[5] Mumtaz R, Zaidi SMH, Shakir MZ, Shafi U, Malik MM, Haque A, Mumtaz S, Zaidi SAR. Internet of Things (IoT) Based Indoor Air Quality Sensing and Predictive Analytic-A COVID-19 Perspective. Electronics.2021;10(2):184.

[6] Sun S, Zheng X, Villalba-Díez J, Ordieres-Meré J. Indoor Air-Quality Data-Monitoring System: LongTerm Monitoring Benefits. Sensors. 2019;19(19):4157.

[7] Cho JH. Detection of Smoking in Indoor Environment Using Machine Learning. Applied Sciences. 2020;10(24):8912.

[8] González E, Casanova-Chafer J, Romero A, Vilanova X, Mitrovics J, Llobet E. LoRa Sensor Network Development for Air Quality Monitoring or Detecting Gas Leakage Events. Sensors. 2020;20(21):6225.

[9] Benammar M, Abdaoui A, Ahmad SHM, Touati F, Kadri A. A Modular IoT Platform for Real-Time Indoor Air Quality Monitoring. Sensors. 2018;18(2):581.

[10] Rajkumar N, Rajendra AB, Vinod V. H2M communication for Home Appliances Automation using Android Applications. Procedia Computer Science. 2020;167:2561-2569. 